\title{
La noción de condicionamiento en la ciencia del comportamiento y el psicoanálisis: discusión entre teorías
}

\author{
Conditioning concept in Behavior Science and Psychoanalysis: discussion between theories \\ Jesús Cisneros-Herrera ${ }^{a}$, Gelacio Guzmán-Díaz $^{b}$
}

\begin{abstract}
:
In this essay, psychoanalysis and behaviour science approaches are presented in relation to their relevance as theoretical alternatives in the field of psychology. Each author argues in support of each approach. Against the general opinion, psychoanalysis does not oppose to basic notions of classical and operant conditioning. If we stick to Freudian theory, both kinds of conditioning are included, for example, in trauma theory and pleasure principle. However, Freud incorporated other concepts that make more complex these notions and break the linearity through which they have been studied experimentally. Behavior science is another proposal to put order in the field of psychology relying on psychological behavior. In tries to reformulate phenomena that are usually associated to cognition and mind. Finally, questions are asked, which psychology should try to answer.
\end{abstract}

Keywords:

Psychoanalysis, behavior science, interbehaviorism, philosophy of science

\section{Resumen:}

En este ensayo, se presentan planteamientos del psicoanálisis y la ciencia del comportamiento respecto de su pertinencia como opciones teóricas en el campo de la psicología. Cada autor argumenta en favor de una y otra. El psicoanálisis, contrario a lo que suele pensarse, no se opone a las nociones básicas de condicionamiento clásico y operante del conductismo. Si nos atenemos a la teoría freudiana, ambas están presentes, por ejemplo, en la teoría del trauma y el principio de placer. Sin embargo, Freud incorporó otros conceptos que complejizan y rompen la linealidad con que estas nociones se han estudiado experimentalmente. La ciencia del comportamiento es otro intento de poner orden en el campo de la psicología basándose en la conducta psicológica. Intenta hacer una reformulación de fenómenos que suelen asociarse con la cognición y la mente en términos interconductuales. Al final, se plantean preguntas que la psicología debería responder.

\section{Palabras Clave:}

Psicoanálisis, ciencia del comportamiento, interconductismo, filosofía de la ciencia

\section{Introducción}

Normalmente, diversidad implica riqueza en cualquier ámbito de la vida: diversidad cultural, diversidad de flora y fauna, diversificación de las inversiones financieras. La diversidad facilita el movimiento, la transformación, la complejización, el desarrollo. El terreno de las ideas y el conocimiento no escapa a esta situación; de hecho, el intento de aniquilar la diversidad para mantener una visión única del mundo, de la naturaleza y del ser humano ha sido una constante en la historia de inmovilizar el estado de cosas por así convenir a los sectores privilegiados y dominantes de las sociedades. Sin embargo, al pensar en la diversidad de las propuestas teóricas en el campo de la psicología, no se observa este factor dinámico que impulse los intercambios y el refinamiento de cada propuesta. En psicología, por el contrario, al parecer del primer autor, los extravíos "teóricos" abundan sin hacer contribuciones serias a la comprensión de los fenómenos psicológicos. Los intentos de "integrar" teorías sólo se suman a la infinidad de propuestas que se pierden en la especificidad de lo concreto, lo aparente.

En este texto, el primer autor argumenta que la teoría psicoanalítica es la que tiene mayor profundidad y

\footnotetext{
a Universidad Autónoma del Estado de Hidalgo, Escuela Superior de Atotonilco de Tula, ORCID: 0000-0003-1370-8086, Email: jesus_cisneros@uaeh.edu.mx

${ }^{\mathrm{b}}$ Universidad Autónoma del Estado de Hidalgo, Escuela Superior de Atotonilco de Tula, ORCID: 0000-0003-4869-8658, Email: gelacio_guzman@uaeh.edu.mx
} 
amplitud, y que las otras así llamadas grandes corrientes teóricas disponen de un aparato conceptual muy limitado y superficial. En muchos casos, estas otras corrientes ni siquiera se contradicen al psicoanálisis, como a veces se da por hecho y se esgrime como argumento para desacreditar la teoría freudiana. Es decir, no hay necesidad de "integrar" al psicoanálisis con otras teorías llamo así a los modelos humanista y cognitivo conductual por mero convencionalismo, puesto que no las considero verdaderas teorías- porque éstas no cubren un terreno ajeno al del psicoanálisis.

Por su parte, el segundo autor argumenta en favor de la ciencia del comportamiento, la cual posee un objeto de estudio propio y aspira a la objetividad.

\section{Psicoanálisis}

\section{Filosofía de la ciencia}

Imre Lakatos, filósofo de la ciencia, sostiene que una teoría sólo debe ser sustituida cuando otra explique lo mismo que su predecesora más fenómenos que ésta no podía explicar (1). Desde este punto de vista, no habría razón para preferir otras teorías por encima de la psicoanalítica, puesto que ninguna puede dar cuenta de todo lo que ésta aborda. Aceptar estas otras teorías equivale a reducir de manera radical el campo de fenómenos considerados objeto de estudio. Es claro que el psicoanálisis ha sido marginado por la psicología académica no porque otras teorías expliquen mejor los mismos fenómenos, sino porque se cuestiona la validez de sus asertos. Karl Popper fue uno de los principales críticos del psicoanálisis: si una hipótesis no se puede poner a prueba en un experimento "objetivo", es decir, si no es falseable, no es científica (1). En realidad, ésta es una idea equivocada de Popper, puesto que en la clínica el analista se formula hipótesis constantemente y en el curso del trabajo se descartan unas, se confirman otras y se reformulan otras más. La dificultad es que el psicoanálisis sigue una lógica propia, que no es la de las ciencias experimentales que recurren al análisis estadístico de los datos.

\section{Psicoanálisis y condicionamiento clásico}

Es bien sabido que el conductismo, hizo su aparición en la psicología como el paradigma que haría de la psicología "una verdadera ciencia", gracias a que sólo admitía como objeto de estudio lo observable. El descubrimiento de Pavlov, denominado en la actualidad condicionamiento clásico, y la ley del efecto formulada por Thorndike y el condicionamiento operante de Skinner constituyeron el corazón de la teoría conductual. Esos dos tipos de condicionamiento podían replicarse una y otra vez, y la referencia a todo concepto "mentalista" era innecesario. Ahora bien, ¿en qué medida estos "descubrimientos" invalidan, se oponen o explican mejor los fenómenos psicológicos?

Recordemos que el condicionamiento clásico consiste en que un estímulo (neutro) que en un principio no produce ninguna reacción en el sujeto, después de ser presentado acompañado de otro que sí produce un efecto (estímulo incondicionado), provoca la misma respuesta (y se convierte en estímulo condicionado). El ejemplo clásico es el trabajo (perverso, vale añadir) de Watson con el pequeño Albert, un niño de menos de un año de edad. Watson le mostraba estímulos que no provocaban miedo al niño -una rata blanca, muñecos de peluche blancos- al mismo tiempo que un sonido muy fuerte que, por sí solo, provocaba llanto y miedo en el niño. Después de cierto número de veces, Albert lloraba con sólo ver la rata o el muñeco de peluche. Las propiedades del estímulo incondicionado (ruido) fueron transferidas a un estímulo neutro (rata, muñeco) para convertirlo en estímulo condicionado. Con esta esquematización, los estímulos externos parecían bastar para explicar la conducta y, en este caso, la fobia del niño.

El descubrimiento de Pavlov fue en 1906 y el experimento de Watson, en 1912. En 1895, Sigmund Freud había redactado un texto metapsicológico, bautizado a posteriori como Proyecto de psicología, en el que presentó el caso de Emma, quien sufría de una fobia a ir a las tiendas sin compañía. Si alguien iba con ella, aunque fuera un niño pequeño, ella podía entrar sin problemas a las tiendas. Emma decía que se avergonzaba del vestido que trajera puesto. La angustia proviene de visitar sola una tienda y se asocia en lo consciente la posibilidad de que los demás se burlen de cómo va vestida. Podríamos ver en la tienda un estímulo condicionado que quedó asociado a una experiencia angustiante $\mathrm{y}$, por eso, ahora despierta la angustia en Emma. La joven ubica el inicio de esa reacción el día que visitó una tienda donde había dos dependientes; se rieron entre ellos y ella pensó que se reían de su vestido. Eso provocó angustia en ella y se marchó; desde entonces no puede ir sola a una tienda.

Quedarse con lo observable, lo evidente, habría supuesto que Freud considerara que la risa de los dependientes provocó la fobia de Emma y que ahora, cada vez que va sola a una tienda, revive la experiencia de sentir que se burlan de ella por su vestido. Aún sin conceptualizar el inconsciente tal como lo conocemos ahora, Freud exploró más la historia de esta joven hasta dar con otro suceso de cuando apenas tenía 8 años de edad. En esa ocasión entró a una tienda y el dependiente lo tocó la región genital encima del vestido, y ella salió corriendo. Otro día regresó como si quisiera repetir la experiencia, pero luego se arrepintió y no volvió más. De esa escena, recuerda la risa del dependiente mientras la tocaba y recuerda su vestido. Entonces, el ataque sexual que sufrió en esa ocasión transfirió su efecto angustiante al hecho de ir sola a una tienda. El mismo esquema del condicionamiento clásico se aplica a la génesis del síntoma fóbico de Emma. El ataque (estímulo incondicionado) provoca una reacción de huida (respuesta incondicionada), pero el lugar en que tuvo lugar (estímulo neutro) años después provoca la misma reacción de huida y evitación; entonces, el estímulo neutro se convierte en estímulo condicionado. La idea que sostiene el primer autor es que el psicoanálisis no se opone a la noción de condicionamiento -clásico u operante-, sino que lo complejiza para hacer justicia a los procesos psíquicos. En el caso del condicionamiento clásico, es claro que no hay contradicción, el esquema del condicionamiento clásico cabe en el modelo 
psicoanalítico, pero éste es mucho más amplio. En su afán de hacer ciencia a la psicología, el conductismo deja fuera, de un modo casi delirante, la propia psique. En las antípodas de lo absurdo, los conductistas desacreditaron a la psicología "mentalista", es decir, rechazaron el estudio de la psique.

Freud no se quedó en el plano de lo evidente, de los estímulos, sino que profundizó en la dialéctica que se da entre lo externo y lo interno. En última instancia, la noción de que las consecuencias de una conducta hacen que ésta tenga mayores 0 menores probabilidades de repetirse en el futuro no es ninguna novedad. Hace miles de años, eso ya se sabía; prueba de ello es el código Hammurabi, donde se establecen los castigos para determinadas conductas. Desde entonces se sabía que los castigos eran necesarios para erradicar las conductas indeseables. La ciencia no consiste en describir lo obvio, como lo hace el conductismo, sino en buscar las fuerzas que subyacen en las manifestaciones fenoménicas (2). La atracción entre los cuerpos como función de su masa y la distancia que los separa no es producto de la mera observación, sino del pensamiento que transforma el conocimiento sensible en teoría científica. La teoría científica implica una ruptura con la observación. Por eso, Freud es más científico que cualquier conductista: él va tras el psiquismo humano y busca la manera de acercarse a él. La observación rigurosa es su punto de partida, pero infiere la dinámica psíquica que está detrás de lo que observa.

¿Qué encuentra entonces Freud en el caso de Emma? En primer lugar, procesos de asociación, tal como hablaban de ellos los empiristas ingleses. El ataque del dependiente, su risa y el vestido de la escena infantil. La temporalidad se incluye en el esquema freudiano, puesto que esta escena está 6 años separada de la que origina el síntoma fóbico, en la que vuelven a conjuntarse los mismos estímulos excepto por el ataque sexual: dependiente, su risa, el vestido. De este modo, Freud (3) hace estallar los modelos de la psique que hasta entonces había con el concepto de Nachträglichkeit, la resignificación del antes por el después, según el cual dos momentos distintos se superponen para dar lugar a una producción nueva: el síntoma en este caso.

Pero ahí no termina la genial aportación de Herr Doktor Freud, puesto que encuentra que dentro del espacio psíquico hay un doble plano que hace más complejo el entramado de los procesos: consciente e inconsciente. El recuerdo de la primera escena no era consciente en Emma, sino hasta que Freud comenzó a indagar en su caso. $Y$ aún después de recordarla, hay algo más que permanece oculto. Entre la primera escena y la segunda, hay un reconfiguración del psiquismo infantil al psiquismo adolescente. Es decir, la primera escena tiene lugar durante la infancia de Emma, mientras que el inicio del síntoma ocurre cuando ya es adolescente. Preguntarnos por las diferencias entre ambas etapas nos lleva a otra pregunta: ¿qué es lo que realmente produce angustia en Emma? ¿El ataque?

Nuevamente, pensar que el solo ataque produjo angustia es quedarnos en el nivel de lo evidente, pero, escuchando con atención las palabras de Emma, Freud encontró que había algo más. Emma dijo que, antes de sentirse angustiada, pensó que uno de los dependientes era atractivo. El ataque que sufre en esta segunda escena no es el de un dependiente, sino de su propia sexualidad que, en la adolescencia, irrumpe para desestabilizar el equilibrio psíquico alcanzado en la etapa de latencia. Cuando Emma dijo que había regresado al lugar del ataque sexual como si quisiera volver a sentir la estimulación, hacía referencia a un placer sexual que para la niña era irrepresentable, así que quedó en una especie de limbo psíquico hasta que llegó la adolescencia. En el momento de ver a un dependiente que le gustaba, el placer sexual que sintió en el ataque se conecta con la sexualidad genital que llega en la adolescencia y es el embate pulsional lo que provoca angustia. El ataque pulsional queda asociado con estar sola en una tienda, lo cual le permite recurrir a la evitación o la huida para mantener alejada de sí la angustia concomitante.

Como se puede ver, el condicionamiento clásico es una noción implícita en la teoría freudiana, pero en el contexto de una dinámica psíquica en que intervienen otros aspectos sumamente importantes, como la separación entre lo consciente y lo inconsciente, y la vida pulsional.

\section{Psicoanálisis y condicionamiento operante}

Los conceptos que intervienen en el condicionamiento operante son conducta y consecuencias, es decir, qué sucede después de realizar cierta conducta. Las consecuencias pueden ser positivas o negativas en tanto que refuerzan o debilitan la aparición de la conducta. El estímulo que fomenta una conducta se denomina reforzador; el que la extingue, castigo. Reforzadores y castigos pueden presentarse de manera negativa, es decir, la desaparición de un estímulo negativo es un reforzador, mientras que la desaparición de un estímulo positivo es un castigo. Hipotéticamente, la conducta puede controlarse si se controlan las consecuencias. Los procesos psicológicos no importan en este modelo conductista. ¿Cómo se percibe el estímulo? ¿Cómo se reconoce como positivo o negativo? ¿Cómo se planea y ejecuta la conducta? Todas éstas son preguntas que carecen de importancia para el conductista.

¿El psicoanálisis está en contra de esta noción de reforzamiento o extinción de conductas? No. En 1911, Freud publicó Formulaciones sobre los dos principios del acaecer psíquico, donde trata el principio de placer y de realidad (4). El principio de placer rige el funcionamiento psíquico, el placer es la búsqueda primordial y última del individuo. Placer es un concepto relacionado con el aspecto económico de la metapsicología, consiste en la reducción de la tensión interna que se produce cuando se acumula en exceso energía en el espacio psíquico. El hambre, por ejemplo, produce tensión; el llanto y el pataleo del bebé son maneras de descargarla. La leche materna cancela temporalmente la fuente de tensión. El bebé no tiene la posibilidad de actuar y buscar el placer por sí solo, pero en cuanto su capacidad física aumenta, su conducta estará dirigida a ese fin y a evitar el displacer y el dolor.

No parece haber incompatibilidad entre condicionamiento operante y principio de placer; son dos formas de denominar una tendencia constante en la conducta. Sin embargo, Freud postuló, además, el principio de realidad 
como sucesor del de placer, en el cual, contrario a lo que sugiere el nombre, no se renuncia al placer, sino que su búsqueda se somete a las condiciones materiales y, mucho más importante aún, a las prohibiciones y normas culturales. El individuo sigue buscando placer, pero la estructuración del aparato psíquico por medio de distintas instancias -ello, yo y superyó- hacen que el placer se complejice, pues cada una de ellas encontrará placer en cosas que a otra pueden producir displacer. Así, la pulsión sexual de un hombre casado, puede dirigirse a una mujer que no es su esposa, pero sus valores morales le indican que la infidelidad es reprobable, inadmisible. La satisfacción pulsional dañaría la imagen narcisizada del yo, de modo que el placer sexual provocaría displacer en el yo: lo que es placer para una instancia, es displacer para la otra (5).

Con estos elementos, Freud inauguró un campo de estudio, un método de investigación, una teoría y un método de tratamiento. Abrió posibilidades únicas para entender la psique y la conducta humana que dan cuenta de su enorme complejidad.

\section{Ciencia del comportamiento}

El enfrentamiento entre conductismo y psicoanálisis parece un tema inevitable en cualquier estudioso de la psicología. El propio Skinner (6) en su obra Acerca del conductismo desarrolla una interpretación operante de los mecanismos de defensa propuestos por Freud. Dicho ejercicio, al parecer del segundo autor, resulta poco acertado. Tanto psicoanálisis como conductismo resultan dos paradigmas que implican dos juegos de lenguaje distintos (véase Wittgenstein, 7). Ambos se enmarcan dentro de lo que se conoce como "Psicología", sin embargo, las dos posturas podrían pertenecer sin problemas a disciplinas independientes fuera de la psicología.

Psicoanálisis y Ciencia del Comportamiento (en donde se incluyen las teorías de condicionamiento clásico, condicionamiento operante, teoría interconductual, teoría de marcos relacionales, entre otras teorías de la conducta) poseen algo distintivo que otros paradigmas incluidos en la llamada "Psicología" no poseen, a saber, bases históricas, epistemológicas y filosóficas que fundamentan su existencia. Y la experimentación, en el caso de la ciencia del comportamiento. Asimismo, los objetos de estudio y los alcances que estas dos disciplinas poseen difieren entre ellos y de las otras "psicologías", lo que hace su comparación poco relevante e incluso innecesaria.

Probablemente el lector quedaría inconforme si esta discusión no se llevara a cabo debido a la justificación anteriormente brindada. Así que defenderé (segundo autor) a la Ciencia del Comportamiento como un paradigma que permita hacer del estudio de lo psicológico algo científico.

\section{Filosofía de la ciencia}

¿Qué es la ciencia?, es la pregunta clave que da inicio a la llamada filosofía de la ciencia. A partir del siglo pasado surgen varios filósofos de la ciencia (ej. Lakatos, Popper, Kuhn, Feyerabend, Carnap, Russell, Bunge, entre otros). Los filósofos de la ciencia se encargan de describir y sistematizar la práctica científica. Es decir, explicitan la caracterización de cómo debería ser una ciencia pero no imponen o establecen las reglas de lo que es o no científico. Curiosamente describen las prácticas científicas sin participar en ellas, ya que muchos de ellos no son científicos.

La ciencia es lo que los científicos hacen. No hay reglamentos, manuales o guías para hacer ciencia. A posteriori, uno podría (como lo hacen los filósofos de la ciencia) describir pasos, características, principios, reglas, instrucciones, procedimientos, técnicas, etc., de lo que hacen los científicos, pero estas prácticas son convencionalidades, y como tales son modificadas por sus propios practicantes.

La práctica científica desde hace siglos ha sido ganada por los físicos, es decir, si una disciplina pretende alcanzar el estatus de ciencia debe realizar actividades análogas al físico. Fue así como surge el primer laboratorio de psicología liderado por Wilhelm Wundt, que propiamente era más un laboratorio de psicofísica.

De acuerdo con Ribes (8), la psicología puede alcanzar un estatus de ciencia básica si tiene un objeto de conocimiento compatible con los criterios y modos en que operan las demás ciencias y, además, si dicho objeto de conocimiento no se yuxtapone ni se reduce a los objetos de conocimiento de otras ciencias ya establecidas.

Por tanto, si Popper u otros filósofos de la ciencia critican y/o niegan el estatus científico del psicoanálisis como ciencia es únicamente por no seguir las prácticas científicas vigentes. Entonces, si el psicoanálisis 0 cualquier otra psicología pretenden pertenecer al grupo de las ciencias pues debe adoptar las convencionalidades al igual que todos. $Y$ no son imposiciones son formas de vida.

\section{Psicoanálisis y condicionamiento clásico}

Watson (9) publica un artículo llamado "La psicología desde el punto de vista conductista". Decir que su proyecto de ciencia psicológica era atribuible a una psicología observable es demeritar sus alcances. Watson manifestaba al conductismo como una ciencia natural cuyo objetivo es el control y predicción de la conducta utilizando la experimentación para lograr objetividad. Es decir, Watson quería hacer de la psicología una ciencia siguiendo las convencionalidades del momento.

Pavlov como bien se menciona resulta fundamental para las ambiciones de Watson, es decir, para una ciencia psicológica, puesto que de manera sistematizada se estudia el comportamiento psicológico. Pavlov (10) observa que los organismos respondemos a objetos cuyas propiedades físicas por si mismas no provocan una reacción fisiológica en el animal, es decir, las respuestas del organismo ya no sólo se explican por su relación biológica sino por una relación funcional, que el llamaría reacciones psíquicas.

Desde los griegos se sabía que los músculos no es lo único que mueve a los organismos, y a lo largo de la historia se han dado explicaciones al respecto. Lo que 
Pavlov hace es sistematizar y mostrarnos un procedimiento, el cual demuestra experimentalmente como se obtienen nuevas relaciones funcionales a partir de la manipulación espacio-temporal de sus componentes. No se adquieren nuevas respuestas sino nuevas relacionales. Para Pavlov, mucho de nuestro comportamiento se adquiere y se mantiene por esta asociación entre eventos.

Pavlov no explica el comportamiento psicológico sino que nos muestra una forma en la que adquirimos un nuevo repertorio de comportamiento. Watson \& Rayner (11) retomarían el pensamiento de Pavlov y estudiaría los principios del condicionamiento clásico con animales humanos, como ya se mencionó con el niño Albert.

El interés de Watson era el estudio de las reacciones emocionales del infante, y cómo el condicionamiento de estas podía estar involucrado en las fobias. Es cierto que el experimento de Watson resulta ser desafortunado en el sentido de que los principios del condicionamiento son aplicables a cualquier reacción emocional, por lo que pudo ver sido el amor o la risa en lugar del miedo lo que se estudiase y los resultados hubieran sido los mismos, es decir, se pueden obtener nuevas relaciones funcionales entre una reacción emotiva y un objeto que en principio no participa de esta relación.

Por otro lado, así como se pueden adquirir nuevas relaciones por condicionamiento también se pueden desaparecer. Watson no pudo llevar a cabo un procedimiento de inhibición de la respuesta debido a que Albert cambio de hogar. Sin embargo, su reacción pudo haberse extinguirse por la falta de asociación entre los estímulos que la provocaban.

Cabe insistir que la importancia del condicionamiento clásico radica en la explicación de cómo adquirimos cierto repertorio psicológico, en donde lo biológico es condición necesaria pero no suficiente. Además, sus principios no se restringen a unos cuantos organismos, a algunos estímulos o a algunas respuestas. Sino que por el contrario sus límites se expanden a cualquier respuesta de un organismo con sistema nervioso y a cualquier objeto funcionalmente pertinente.

Como bien se menciona la ciencia no describe "lo obvio", de acuerdo con Ribes (12) las ciencias empíricas tienen dos niveles de funcionamiento. En un primer nivel, las ciencias trabajan en un plano descriptivo, intentando sistematizar las regularidades que se observan, de manera natural, en los fenómenos de su interés. Los fenómenos observados se comparten con los que se vive comúnmente en la vida cotidiana. Sin embargo, aunque el universo de observación seleccionado es el mismo, el criterio de observación no lo es.

Por ejemplo, en el caso de la biología, se hacen taxonomías de los seres vivos a partir de su estructura y sus funciones. Desde muchos años antes de que la biología existiera cualquier persona sin conocimientos de la misma, podía reconocer las diferencias entre un gato y una bacteria. Es a partir de la observación, las hipótesis, la experimentación y los resultados que la ciencia se aleja del sentido común y del lenguaje ordinario para construir un lenguaje técnico propio de una ciencia.

En un segundo nivel, de acuerdo con Ribes (12) surge el lenguaje técnico, que trasciende el de la observación común. Los criterios de observación son muy selectivos y no se basan en las observaciones comunes. Son observaciones guiadas teóricamente y, en esa medida, su descripción se realiza en un lenguaje abstracto que no hace referencia a particulares.

Siguiendo con el ejemplo de la taxonomía de los seres vivos, una vez que se estudian las propiedades relevantes de dichos eventos se evalúa la pertinencia de establecer relaciones analíticas con base en criterios teóricos explícitos. Es así como el gato y la bacteria, ya no sólo se clasifican con base en lo "obvio" sino que se catalogan como eucariotas y procariotas con base en la comparación de las secuencias del gen que codifica para el ARN ribosomal.

Regresando al caso de la psicología, tanto el condicionamiento clásico como el operante no están interesados en lo "obvio", están interesados en encontrar lo común en lo particular. Es decir, explicar mediante su modelo cualquier comportamiento psicológico. Si el caso de Emma o cualquier otro caso psicoanalítico, es posible explicarlo en el lenguaje del condicionamiento, el objetivo científico de estas teorías se ha logrado.

El poder explicativo de las teorías del condicionamiento pretende dar cuenta de cualquier fenómeno psicológico. Más tarde Pavlov propondría el estudio del lenguaje como la adquisición de un segundo sistema de señales, lo que sería retomado en los estudios neurológicos del lenguaje de Luria, en el condicionamiento semántico y en la formación de clases de estímulos equivalentes de Sidman. Del mismo modo, es posible reinterpretar en términos el condicionamiento los estudios de formación de conceptos de Vygotsky y los estudios de etapas de desarrollo de Piaget, entre otras aproximaciones psicológicas.

\section{Caso Emma: perspectiva interconductual}

En ciencia no se estudian eventos particulares, es decir, ni Emma, ni Albert, ni cualquier otro caso relacionado con fobias es por si solo importante para el estudio científico. En ciencia se abstraen las propiedades relevantes de cada evento y se evalúa la pertinencia de establecer relaciones analíticas con base en criterios teóricos explícitos (12).

Lo común en el caso de Emma y Albert son las contingencias pavlovianas. Así mismo, el caso completo de Emma podría ser explicado como una cadena muy compleja de repuestas condicionales y operantes, sin embargo, las exigencias explicativas al modelo conductual seguirán siendo las ya expresadas.

Cierto es que la ciencia del comportamiento no puede dar respuestas a muchas de las preguntas psicoanalíticas debido a que son preguntas que parten de una lógica dualista. Igualmente sería un sinsentido que el psicoanálisis nos explicara los eventos reforzantes que mantienen el comportamiento de Emma. Cuando se dice que el condicionamiento no profundiza en la dialéctica entre lo externo y lo interno, es debido a que dentro del modelo conductual tal dicotomía no tiene cabida. Sin embargo, no se rechaza el estudio de la dialéctica que pudiese tener una persona consigo misma a forma de soliloquio y cómo este participa dentro del fenómeno psicológico. 
Kantor (13) desarrolla la psicología interconductual, en la que el comportamiento implica la interacción entre la actividad de un organismo o individuo y aquella que es desplegada por los objetos, eventos $u$ otros organismos e individuos del ambiente, regulada por factores disposicionales tanto organísmicos como ambientales y posibilitada por diferentes medios de contacto.

Dentro de la perceptiva interconductual Ribes y López (14) desarrollan una teoría de la conducta, que consiste en una taxonomía de funciones estímulo-respuesta que puede ser de cinco tipos progresivamente más complejos e inclusivos: (a) contextual; b) suplementaria; c) selectora; d) sustitutiva referencial; y e) sustitutiva no referencial). Dicha clasificación depende, entre otros elementos, del desligamiento funcional, que describe la relativa autonomía de la actividad del organismo o individuo respecto de las propiedades concretas de los eventos y de las características temporo-espaciales que las definen. Los tres primeros niveles se consideran intrasituacionales en el sentido de que ocurren en una situación espaciotemporal determinada. Los últimos dos niveles implican eventos de estímulo y de respuesta lingüísticos cuya arbitrariedad respecto de la situacionalidad permite trascenderla parcial o totalmente.

Con los datos que tenemos podríamos considerar el caso de Emma como un ejemplo de sustitución referencial en el que las funciones de referidor o mediador y de referido o mediado descansan en sólo una persona.

Las interacciones sustitutivas referenciales consisten en un hablante, gesticulador o escritor (mediador) que modifica mediante una intervención lingüística las relaciones de contingencia prevalentes en una situación. El mediado debe comportarse en correspondencia con la conducta del mediador y con las contingencias que introduce en la situación como contingencias referidas (15).

Dada la inclusividad de la taxonomía de Ribes y López (14), los niveles más complejos incluyen a los menos complejos por lo que las contingencias pavlovianas que se describen en el caso de Emma corresponden a interacciones intrasituacionales dentro de una interacción sustitutiva referencial. La función sustitutiva referencial transciende tiempo y espacio por lo que una interacción psicológica puede comenzar hoy y culminar dentro de 5 , 10 o más años.

En el caso Emma, lo que sugiere que podría ser un caso de sustitución referencial, es el lenguaje y "pensamientos" que tiene con ella misma, es decir, funcionalmente toma un papel de mediador y mediado acerca de la situación acontecida. Esto lo deducimos, por ejemplo, en frases como: "Emma dijo que, antes de sentirse angustiada, pensó que uno de los dependientes era atractivo", "Cuando Emma dijo que había regresado al lugar del ataque sexual como si quisiera volver a sentir la estimulación".

Si revisamos con atención el caso de Emma y otros casos descritos por Freud, como el caso del pequeño Hans que también involucraba una fobia, podemos encontrar características comunes, siempre hay un referente, un referido y un referidor, a veces el referidor es el mismo paciente o Freud, o como en el caso de Hans, el referidor es el padre.
Para el interesado en el psicoanálisis, la interpretación del caso de Emma a partir de la taxonomía del comportamiento propuesta por Ribes y López (1985), pudiese resultar poco clarificadora e igual de útil que la del condicionamiento clásico. Sin embargo, recordemos que en ciencia no tratamos particulares, buscamos orden y regularidades y sobretodo investigación experimental. No podemos estudiar experimentalmente el caso de Emma, pero si podemos estudiar la sustitución referencial y lo que obtengamos de esta pesquisa nos dirá más acerca de Emma, Hans, y cualquier otro caso particular.

\section{Comentarios finales}

De acuerdo con el primer autor, la psicología tendría que hacer una autocrítica respecto de la forma desmesurada de proponer y adoptar teorías que no constituyen un verdadero avance científico, lo cual, además, afecta la formación profesional del psicólogo, que se ve expuesto a un sinfín de teorías que lo fuerzan a memorizar en vez de comprender. La teoría psicoanalítica posee una compleja red conceptual en la que conceptos de otras teorías, como lo hemos visto aquí en el caso del conductismo, que la convierte en la más sólida, amplia y profunda.

En cambio, el segundo autor sostiene la idea de que psicoanálisis y ciencia del comportamiento son dos disciplinas independientes, cada una con su objeto de estudio y sus fundamentos epistemológicos. Estas diferencias hacen que, cuando menos en algunos casos, la discusión entre ambas sea impensable.

Surgen diversas preguntas: ¿ciencia del comportamiento y psicología son dos disciplinas diferentes?, ¿puede equipararse psicoanálisis a psicología?, ¿cuáles son las aportaciones exclusivas de cada una para comprender al ser humano? Estas preguntas tendrías que responderse más allá de recurrir al argumento de las formalidades y la convenciones, y aventurarse en el terreno de la cosa-ensí kantiana.

\section{Referencias}

1. Pérez Tamayo R. ¿Existe el método científico? México: Fondo de Cultura Económica; 1993. (La ciencia para todos).

2. Braunstein NA. ¿Cómo se constituye una ciencia? En: Psicología: ideología y ciencia. México: Siglo XXI; 1975. p. 7-20.

3. Freud S. Proyecto de psicología. En: Obras completas. Buenos Aires, Argentina: Amorrortu; 1895.

4. Freud S. Formulaciones sobre los dos principios del acaecer psíquico. En: Obras completas. Buenos Aires, Argentina: Amorrortu; 1911.p. 217-32.

5. Freud S. La represión. En: Obras completas. Buenos Aires, Argentina: Amorrortu; 1915. p. 135-52.

6. Skinner, B. F. About behaviorism. New York: Random House; 1974.

7. Wittgenstein, L. Philosophical investigations. Oxford: Basil Blackwell.; 1953.

8. Ribes, E. Las psicologías y la definición de su objeto de conocimiento. Rev Mex Análisis Conducta. 2000;26:367-83.

9. Watson, J. B. Psychology as the behaviorist views it. Psychol Rev. 1913;(20):158-77.

10. Pavlov, I. Conditioned reflexes: An investigation of the physiological activity of the cerebral cortex. England: Oxford; 1927.

11. Watson, J. B. R R. Conditioned emotional reactions. J Exp Psychol. 1920;3:1-14.

12. Ribes, E. La psicología como ciencia básica. ¿Cuál es su universo de investigación? Rev Mex Investig En Psicol. 2009;1(2):7-19. 
13. Kantor, J. R. Interbehavioral psychology: A sample of system construction. Granville: Principia Press; 1959.

14. Ribes, E. L F. Teoría de la conducta. México: Trillas; 1985.

15. Ribes, E. Las funciones substitutivas de contingencias. En: La función substitutiva referencial: análisis histórico-crítico Avances y perspectivas. New Orleans, LO: University Press of the South; 2012. p. 19-34. 